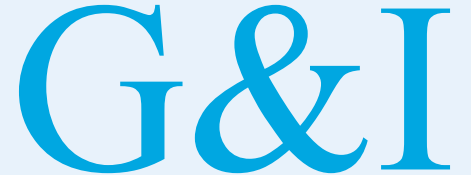

Genomics \& INFORMatics

\section{Review article}

elSSN 2234-0742

Genomics Inform 2020;18(4):e36

https://doi.org/10.5808/GI.2020.18.4.e36

Received: December 3, 2020

Revised: December 10, 2020

Accepted: December 10, 2020

*Corresponding author:

E-mail:wykim82@knue.ac.kr

\title{
The ceRNA network of IncRNA and miRNA in lung cancer
}

\author{
Danbi Seo ${ }^{1}$, Dain Kim ${ }^{1}$, Yeonsoo Chae ${ }^{1,2}$, Wanyeon $\mathrm{Kim}^{1,3^{*}}$ \\ 'Department of Science Education, Korea National University of Education, Cheongju \\ 28173, Korea \\ ${ }^{2}$ Department of Science Education, Chungbuk Science High School, Cheongju 28189, Korea \\ ${ }^{3}$ Department of Biology Education, Korea National University of Education, Cheongju \\ 28173, Korea
}

Since lung cancer is a major causative for cancer-related deaths, the investigations for discovering biomarkers to diagnose at an early stage and to apply therapeutic strategies have been continuously conducted. Recently, long non-coding RNAs (IncRNAs) and microRNAs (miRNAs) are being exponentially studied as promising biomarkers of lung cancer. Moreover, supportive evidence provides the competing endogenous RNA (ceRNA) network between IncRNAs and miRNAs participating in lung tumorigenesis. This review introduced the oncogenic or tumor-suppressive roles of IncRNAs and miRNAs in lung cancer cells and summarized the involvement of the IncRNA/miRNA ceRNA networks in carcinogenesis and therapeutic resistance of lung cancer.

Keywords: competing endogenous RNA, long non-coding RNA, lung tumorigenesis, miRNA, therapeutic resistance

\section{Introduction}

Lung cancer is a malignant disease of the lungs and accounts for a large proportion of all cancer-attributable deaths [1]. Lung cancer is classified as non-small cell lung cancer (NSCLC) or small cell lung cancer (SCLC), and NSCLC accounts for around $80 \%$ of all lung cancers [2]. Lung cancer is one of the most difficult cancers to diagnose at an early stage because it has few initial symptoms [3]. However, early diagnosis and surgical treatment provide the best strategy in terms of increasing survival rates [4], and thus, medically applicable biomarkers are required for early diagnosis.

Only 2\% of RNA transcribed from human DNA encodes proteins, and the remaining 98\% is referred to as non-coding RNA (ncRNA) [5]. Although not translated into proteins, ncRNAs perform various functions within cells $[6,7]$ and have potential use as biomarkers for the diagnosis of lung cancer. Long non-coding RNAs (lncRNAs) and microRNAs (miRNAs) are representative examples of ncRNA [8,9] and are being actively studied as potential biomarkers. Furthermore, accumulating evidence indicates competing endogenous RNA (ceRNA) networks of lncRNA and miRNA affect carcinogenesis. This review addresses the association between the ceRNA network of lncRNAs and miRNAs and the development of lung cancer and provides an overview of the effect of this network on the survival, proliferation, motility, and radiation and drug resistances of lung cancer cells. 


\section{The Roles of miRNAs and LncRNAs in Lung Cancer}

MiRNA is around 22 nucleotides in length and an evolutionarily conserved type of ncRNA that suppresses gene expression by interacting directly with DNA and RNA $[10,11]$. In cytoplasm, miRNA inhibits translation by destabilizing and causing the degradation of mRNA, regulating transcriptional stability in the nucleus, and recruiting epigenetic remodeling factors to induce gene silencing [12]. MiRNA is involved in various biological processes such as cell cycle development, cell differentiation, and does so by regulating the expressions of target genes. Furthermore, abnormal miRNA expression is associated with many diseases including cancer [13].

In particular, miRNA may be directly involved in carcinogenesis. Genomic instability is one of the hallmarks of cancer and facilitates tumorigenic process [14]. Certain miRNA genes are placed in chromosomes which are susceptible to damage and mutation, and physical disruption of these miRNAs may be responsible for a wide range of abnormalities in the expressions of genes that play critical roles in the cell cycle, DNA repair, and apoptosis. In cancer, miRNAs are classified as oncogenic miRNAs (onco-miRs) or tumor-suppressive miRNAs. Onco-miRs target tumor-suppressive mRNAs, CDK inhibitors, and pro-apoptotic members of the Bcl2 family, and thus, promote tumor growth and anti-apoptotic signaling [15]. In contrast, tumor-suppressive miRNAs disrupt the expressions of oncogenic mRNAs such as those of cyclins, CDKs, and genes that are directly and indirectly involved in growth factor-mediated signaling pathways and inhibit cell proliferation and survival [16]. Various miRNAs have been shown to be involved in cell proliferation and death in lung cancer. In NSCLC cells, miR21 affected cell growth and invasion by targeting the PTEN transcript [17], whereas miR-451a regulated the migration and invasion of lung cancer cells by targeting ATF2 [18]. Interestingly, these miRNAs are potentially involved in the development of lung cancer and can be regulated by interaction with lncRNAs.

LncRNAs, like miRNAs, represent a major group within the ncRNA family. LncRNAs are considerably longer than miRNAs; they contain around 200 nucleotides and are more than $100 \mathrm{~kb}$ long and some have poly A tails $[19,20]$. In cells, lncRNAs play a variety of roles, such as activating signaling pathways, modifying chromatin, and regulating transcription and translation [21]. In particular, lncRNAs can regulate mRNA expression by competing with miRNA in cytoplasm [22]. It was suggested some lncRNAs have sponge-like effects on miRNAs that attenuate the effects of mRNAs, which is referred to as the ceRNA hypothesis [23,24]. In fact, many lncRNAs have miRNA-binding sites that regulate the expressions of genes encoding proteins [25]. LncRNA, which functions as a ceRNA, sequesters miRNA and prevents them regulating the translations of target mRNAs (Fig. 1). In 2014, lncRNA AK048451, which is called cardiac hypertrophy related factor, was first identified as a ceRNA of miR-489 and found to inhibit miR-489 expression by direct binding in a sequence-specific manner [20]. Furthermore, abnormal expressions, mutations, and single nucleotide polymorphisms of lncRNA have been associated with tumor formation and metastasis [26], and accumulating evidence indicates networks of lncRNAs, miRNAs, and mRNAs importantly contribute to the epithelial-to-mesenchymal transition (EMT), onset and progression of cancer $[27,28]$. For example, lncRNAs (MEG3, MIAT, and LINC00115) were found to play important roles in carcinogenesis by regulating miRNA-mRNA networks in lung cancer [29].

\section{Oncogenic LncRNAs Acting as ceRNAs in Lung Cancer}

Oncogenic lncRNAs are generally upregulated in lung cancer cells and tissues and bind directly to tumor-suppressive miRNAs. Direct lncRNA to miRNA binding upregulates the expressions of oncogenic mRNAs (a target of miRNAs), and thus, promotes cancer cell growth and development. Several lncRNAs that function as oncogenes in lung cancer have been identified (Table 1), for example, lncRNA H19 is highly expressed in the A549, H1299, H23, and SPC-A1 lung cancer cell lines, and inhibits miR-200a, miR196b, and miR-29b-3p [30-32]. The interaction between lncRNA H19 and miR-200a (a tumor-suppressive miRNA downregulated in patients with a high lung cancer stage) regulates the expressions of ZEB1 and ZEB2 [30]. LIN28B is a target of miR-196b and can function as a proto-oncogene, and lncRNA H19 can upregulate LIN28B by 'sponging' miR-196b [31]. MiR-29b-3p is involved in the regulation of apoptosis, the cell cycle, and metastasis, and its targeting by lncRNA H19 transforms STAT3 (signal transducer and activator of transcription 3), and thus, promotes the survival and EMT of lung adenocarcinoma cells [32].

MALAT1 (metastasis associated in lung adenocarcinoma transcript 1) is another representative oncogenic lncRNA and is highly expressed in the A549 and H1299 lung cancer cell lines, in which miR-124 is downregulated. MiR-124 is a direct target of MALAT1 and inhibits the expression of STAT3 [33], and the expression of MALAT1 has also been reported to be correlated with the expressions of miR-200a-3p and programmed death-ligand 1 (PD-L1) [34]. MALAT1 acts as a sponge for miR-200a-3p, and thus, increases the expression of PD-L1 (a direct target of miR-200a-3p), inhibits apoptosis, and promotes the metastasis of NSCLC cells 


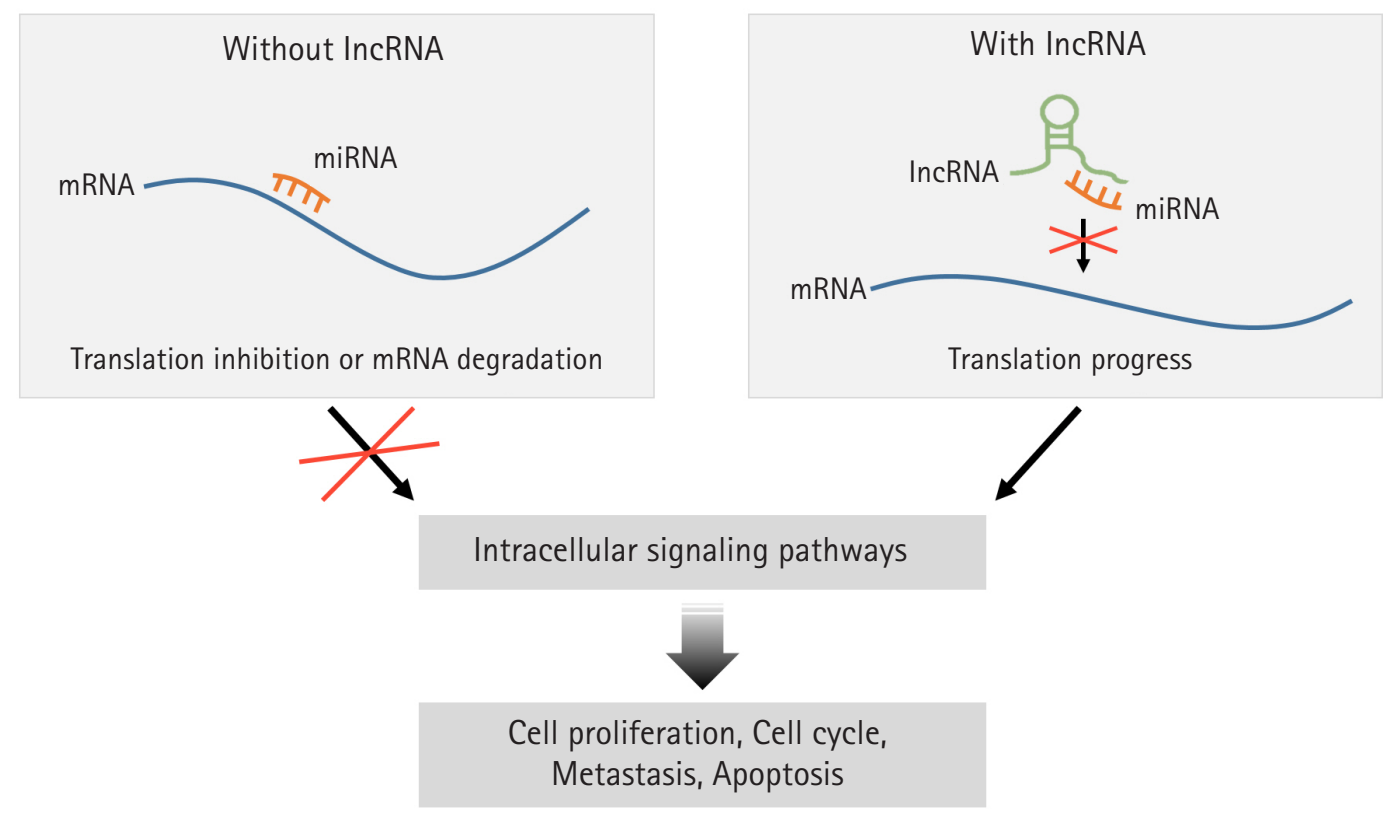

Fig. 1. Principle of the ceRNA interaction between IncRNA and miRNA in cancer. LncRNAs can regulate mRNA expressions by competing with miRNAs. LncRNAs that have miRNA-binding sites regulating the expressions of genes encoding proteins can act as miRNA sponges that attenuate miRNA activity, and thus, lead to translation of target mRNAs. According to intracellular signaling pathways associated by the mRNAs, cell proliferation, cell cycle, metastasis and apoptosis can be promoted in cancer cells. ceRNA, competing endogenous RNA; IncRNA, long non-coding RNA; miRNA, microRNA.

Table 1. Interactions of oncogenic IncRNAs with tumor-suppressive miRNAs in lung cancer

\begin{tabular}{|c|c|c|c|c|c|}
\hline IncRNA & $\begin{array}{c}\text { miRNA } \\
\text { (direct interaction with IncRNA) }\end{array}$ & $\begin{array}{l}\text { The number of } \\
\text { miRNA- binding sites }\end{array}$ & mRNA (target of miRNA) & Effects of IncRNA in cells & Reference \\
\hline \multirow[t]{3}{*}{ H19 } & miR-200a & - & ZEB1, ZEB2 & $\begin{array}{l}\text { Promoting cell proliferation, migration and } \\
\text { invasion }\end{array}$ & [30] \\
\hline & miR-196b & 7mer-m8 & LINC28B & Promoting cell proliferation & [31] \\
\hline & miR-29b-3p & 8mer & STAT3 & $\begin{array}{l}\text { Promoting cell proliferation and metasta- } \\
\text { sis, inhibiting apoptosis }\end{array}$ & [32] \\
\hline \multirow[t]{2}{*}{ MALAT1 } & miR-124 & 7mer-m8 & STAT3 & Promoting cell proliferation & [33] \\
\hline & miR-200a-3p & 7mer-m8 & PD-L1 & Promoting metastasis, inhibiting apoptosis & [34] \\
\hline DANCR & miR-216a & 8mer & EIF4B, JAK2 & Promoting cell proliferation & [35] \\
\hline LINC00336 & miR-6852 & - & CBS & Inhibiting ferroptosis & [36] \\
\hline MNX1-AS1 & miR-527 & - & BRF2 & $\begin{array}{l}\text { Promoting cell proliferation, migration and } \\
\text { invasion }\end{array}$ & [37] \\
\hline LINC00673 & miR-150-5p & - & ZEB1 & $\begin{array}{l}\text { Promoting cell proliferation, EMT, migra- } \\
\text { tion and invasion }\end{array}$ & [38] \\
\hline SNHG4 & miR-98-5p & 7 mer-m8 & CDK6, SALL4 & $\begin{array}{l}\text { Promoting cell proliferation, EMT, } \\
\text { migration and invasion }\end{array}$ & [39] \\
\hline LEF1-AS1 & $\operatorname{miR}-489$ & 7mer-m8 & SOX4 & $\begin{array}{l}\text { Promoting cell proliferation and migration, } \\
\text { inhibiting apoptosis }\end{array}$ & [40] \\
\hline UCA1 & miR-193a-3p & - & ERBB4 & Promoting cell proliferation & [41] \\
\hline SNHG1 & miR-497 & 7mer-m8 & - & $\begin{array}{l}\text { Promoting cell proliferation, migration and } \\
\text { invasion }\end{array}$ & [42] \\
\hline PTAR & miR-101 & - & - & $\begin{array}{l}\text { Promoting cell proliferation, migration and } \\
\text { invasion }\end{array}$ & [43] \\
\hline
\end{tabular}

IncRNA, long non-coding RNA; miRNA, microRNA.

${ }^{a}$ The number of miRNA-binding sites were provided based on the ENCORI database (http://starbase.sysu.edu.cn/index.php) [25]. 
[34]. PD-L1 is an attractive factor in cancer research, and drugs that target it have been shown to improve patient prognoses $[44,45]$.

In addition, lncRNAs such as DANCR, LINC00336, MNX1AS1, LINC00673, SNHG4, LEF1-AS1, UCA1 (urothelial carcinoma-associated 1), SNHG1, and PTAR act as ceRNAs for miRNAs and exhibit oncogenic functions in lung cancer cells. For example, DANCR inhibited miR-216a, and thereby, upregulated EIF4B and JAK2, which are targets of miR-216a [35], and LINC00336 upregulated the expression of cystathionine- $\beta$-synthase (CBS) by competing with miR-6852 [36]. MNX1-AS1 functioned as an oncogene in lung cancer by sponging miR-527, and thus, activating the BRF2 (TFIIB-related factor 2) signaling pathway [37]. LINC00673 acted as a ceRNA by sponging miR150-5p, and indirectly regulating ZEB1 expression [38]. SNHG4 acted as a sponge for miR-98-5p, which can directly target CDK6 and SALL4, and SALL4 is upregulated in lung cancer tissues [39]. LEF1-AS1 is mainly confined to cytoplasm, and binds miR-489 and activates SOX4, which inhibits apoptosis and promotes tumor development and progression [40]. The expression of UCA1 was increased in human NSCLC tissues and associated with poor prognoses [41]. Furthermore, the positive effect of UCA1 on NSCLC cell proliferation was attributed to sponging of miR-193a-3p, and the administration of miR-193a-3p blocked the effect of UCA1 by targeting ERBB4 [41]. Lnc-SNHG1 can accelerate the progression of NSCLC by acting as a sponge for miR-497 [42], and lncRNA PTAR promoted NSCLC cell proliferation, migration, and invasion by sponging miR-101 [43].

Several lncRNAs such as H19, MALAT1, and DANCR act as oncogenes in lung cancer by interacting with miRNAs. Since these IncRNAs are upregulated in lung cancer cells and tissues, and can be used as and are viewed as potential biomarkers for the early diagnosis of lung cancer. Therapies based on the use of ceRNA networks of oncogenic lncRNAs and miRNAs targeting these genes should be useful for the treatment of lung cancer.

\section{Tumor-suppressive IncRNAs acting as ceRNAs in lung cancer}

Unlike lncRNAs that are upregulated in lung cancer cells and tissues and function as oncogenes, tumor-suppressive lncRNAs are generally downregulated in lung cancer cells and tissues and bind directly to onco-miRs. Direct binding of tumor-suppressive lncRNAs and onco-miRs upregulates the expressions of tumor-suppressive mRNAs, a target of onco-miRs, which inhibit cancer cell growth and development. Several lncRNAs have been identified that function as tumor suppressors in lung cancer (Table 2). For example, lnc ADAMTS9-AS2 is downregulated in lung cancer cells and tissues and inhibits the development of lung cancer cells $[46,47]$. This function of lnc ADAMTS9-AS2 is due to direct interaction with miR-223-3p, which regulates the expression of TGFBR3 [46]. Increased lnc ADAMTS9-AS2 expression in lung cancer cells and tissues downregulated mIR-223-3p [46], and as a result, TGFBR3 was upregulated and cancer progression was suppressed [46].

In addition, lncRNAs such as MT1JP, MAGI2-AS3, PLAC2, TINCR, LINC00641, FENDRR (FOXF1 adjacent non-coding developmental regulatory RNA), TRHDE-AS1, and lncRNA-p21 act as tumor suppressors in lung cancer by sponging miRNAs. For example, MJ1JP inhibited the proliferation, invasion, and migration of A549 lung cancer cells, and this inhibition was attributed to Bim upregulation due to the sponging of miR-423-3p [48]. MAGI2-AS3 is downregulated in NSCLC, and its overexpression decreased the proliferative and invasive capacities of NSCLC cells [49]. MAGI2-AS3 sponges miR-23a-3p, and miR-23a-3p directly interacts with PTEN [49]. In another example, low PLAC2 expression predicted poor survival in NSCLC patients, and the overexpression of PLAC2 downregulated miR-21 and upregulated PTEN, a direct target of miR-21 [50]. TINCR acted as a sponge for miR544a and inhibited the proliferation and invasion of lung cancer cells, but miR-544a directly interacted with FBXW7 and reversed TINCR sponging miR-544a [51]. LINC00641 upregulated the expression of PLSCR4 by sponging miR-424-5p, and as a result, inhibited the proliferation and induced the apoptosis of NSCLC cells [52]. The IncRNA FENDRR upregulated TIMP2 (tissue inhibitor of metalloproteinase 2) by directly binding miR-761, an inhibitor of TIMP2 in NSCLC, and suppressed the aggressiveness of NSCLC cells [53]. TRHDE-AS1 inhibited the proliferation and invasion of lung cancer cells by up-regulating KLF4 (a tumor-suppressor) by inhibiting miR-103, and the overexpression of miR-103 reversed the effect of TRHDE-AS1 [54]. Also, IncRNA-p21 had a direct binding site for miR-17-5p, and binding between the two inhibited NSCLC progression [55].

Another tumor-suppressive lncRNA GAS5 (growth arrest-specific transcript 5) inhibits tumor formation in lung cancer by negatively regulating miR-205 expression, and thus, increasing PTEN expression [56]. In NSCLC, GAS5 inhibited the expression of miR-23a, cell proliferation, and invasion and promoted apoptosis [57]. In addition, GAS5 improved the radiosensitivity of NSCLC cells [58]. Radiotherapy kills cancer cells by exposing them to high-energy radiation [59], and greater radiosensitivity of cancer cells is strongly associated with positive treatment results [60].

LncRNAs such as lnc ADAMTS9-AS2, MT1JP, and GAS5 act as tumor suppressors in lung cancer through $\operatorname{lncRNA} / \mathrm{miRNA}$ ceRNA networks, which regulate the expressions of well-known 


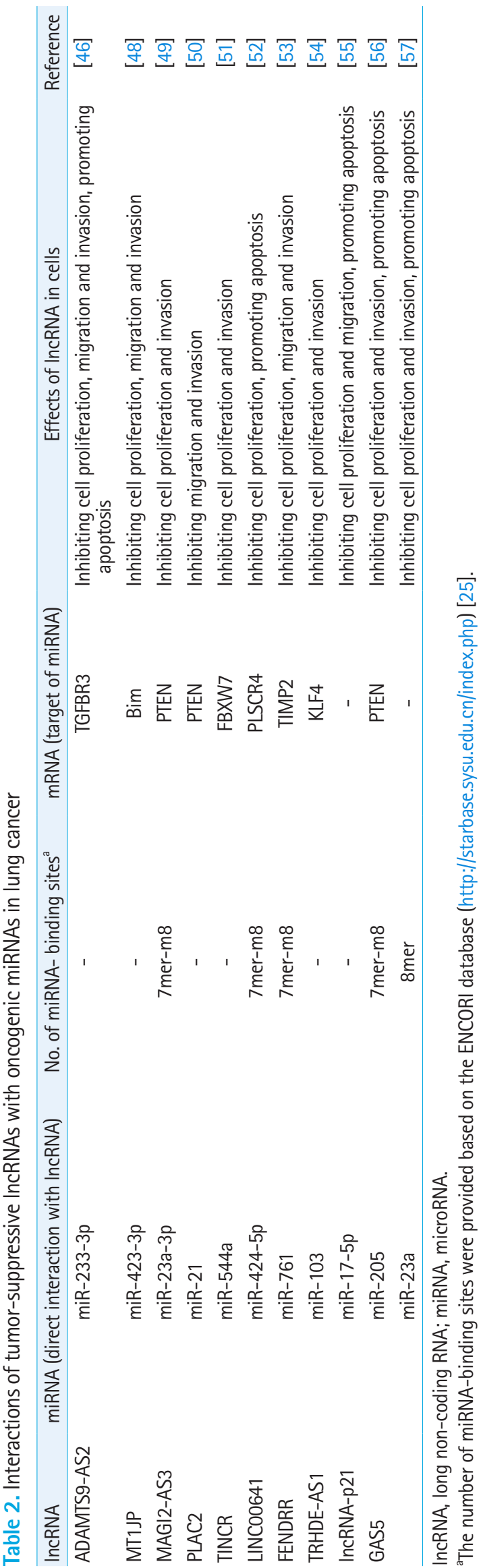

tumor suppressors such as PTEN and TIMP2. Like oncogenic lncRNAs, interactions between tumor-suppressive lncRNAs and onco-miRs may aid early diagnosis and provide gene-targeting therapies for lung cancer.

\section{The ceRNA Roles of LncRNAs in Therapeutic Resistance}

Non-surgical methods of treating lung cancer include radiation therapy and drug therapy. Representative drugs for the treatment of lung cancer include gefitinib and cisplatin. Gefitinib inhibits epidermal growth factor receptor (EGFR) tyrosine kinase by binding to the enzyme's ATP-binding site [61]. Gefitinib sensitivity studies in NSCLC have shown that mutations in the tyrosine kinase domain of EGFR activate the anti-apoptotic pathway [61]. On the other hand, cisplatin kills the fastest growing cancer cells by interfering with DNA replication [62]. The developments of radiation and drug resistance are major obstacles to successful non-surgical cancer treatment. Accordingly, studies are being actively conducted on genes involved in signaling pathways that improve sensitivity to radiation or drugs, and evidence is accumulating that lncRNA/miRNA networks are involved. For example, it was reported LINC00483 silences miR-144 in lung adenocarcinoma, and thereby, increases the radiosensitivity of LTEP-A-2 cell lines [63]. Also, FAM201A lncRNA was found to be highly expressed in NSCLC patients resistant to radiation therapy and function as a ceRNA of miR-370 and increase the expressions of EGFR and HIF-1 $a$ (hypoxia-inducible factor 1 alpha) [64]. FAM201A knockdown suppressed the expressions of EGFR and HIF-1 $\alpha$ and increased the radiosensitivity of NSCLC cells [64]. Furthermore, in NSCLC cells CYTOR (cytoskeleton regulator RNA) lncRNA sponged miR-195 and suppressed radiosensitivity of NSCLC cells in vitro [65].

In a study on drug resistance, overexpression of HOST2 (human ovarian cancer-specific transcript 2) lncRNA inhibited miR621 and increased gefitinib resistance in NSCLC cells due to the upregulation of SYF2 (a direct target of miR-621) [66]. LIN C00460 was highly expressed in gefitinib-resistant NSCLC cells and tissues and upregulated EGFR through miR-769-5p sponging [67]. Furthermore, EGFR upregulation led to gefitinib resistance [67]. In addition, in cisplatin-resistant NSCLC cells, TATDN1 (Homo sapiens TatD DNase domain containing 1) lncRNA downregulated miR-451, which was overexpressed in these cells, and TATDN1 knockdown improved cisplatin sensitivity [68]. Also in cisplatin-resistant NSCLC cells, TATDN1 and TRIM66 (a target of miR-451) gene expressions were positively correlated and TRIM66 was overexpressed [68]. In SCLC cells, LINC00173 
sponged miR-218 and induced cisplatin and etoposide (an SCLC therapeutic) resistance [69].

The lncRNA/miRNA ceRNA network regulates the expressions of several genes that act as oncogenes or tumor suppressors in lung cancer. In several studies, changes in gene expressions by lncRNA/miRNA induced drug and radiation resistance in lung cancer cells, which suggests that the ceRNA network has the potential to contribute to the efficient applications of traditional cancer and gene-targeting therapies.

\section{Conclusion}

Studies on the lncRNA/miRNA ceRNA network in lung cancer are being actively conducted. Direct binding between $\operatorname{lncRNA}$ and miRNA influences cancer progression by regulating the expressions of various mRNAs that act as oncogenes or tumor suppressors. In the ceRNA network, the expressions of lncRNA and miRNA are negatively correlated, lncRNA binding to onco-miRs suppresses tumor progression, whereas its binding to tumor-suppressive miRNAs promotes tumor progression. In this review, we summarize the effects of various lncRNAs that function as ceRNAs of miRNAs in lung cancer.

LncRNAs that function as oncogenes in lung cancer through ceRNA networks include H19, MALAT1, DANCR, LINC00336, MNX1-AS1, LINC00673, SNHG4, LEF1-AS1, UCA1, SNHG1, and PTAR. In contrast, lncRNAs that function as ceRNAs of miRNAs and act as tumor suppressors in lung cancer include ADA MTS9-AS2, MT1JP, MAGI2-AS3, PLAC2, TINCR, LINIC00641, FENDRR, TRHDE-AS1, IncRNA-p21, and GAS5. Furthermore, these lncRNAs confer radiation and chemical resistance in lung cancer.

For example, LINC00483, FAM201A, and CYTOR induce radioresistance by directly binding miRNAs, and HOST2, LINC00460, TATDN1, and LINC00173 induce drug resistance to gefitinib and cisplatin. As such, many lncRNAs contribute to the development of lung cancer in various ways by direct binding miRNAs.

In this review, we summarize the lncRNA/miRNA ceRNA networks that impact lung cancer identified to date, and provide insight into the effects of RNAs not translated into proteins and of the various signaling pathways that act on lung cancer through downstream factors. The lncRNA/miRNA ceRNA network offers a means of discovering biomarkers that enable the early diagnosis of lung cancer and provide guidance regarding gene-specific treatments. In addition, the abilities of lncRNA and miRNA interactions to affect radiation and drug resistance suggests they can be targeted in treatment of resistant patients. We believe improved understanding of lncRNA and miRNA interactions is likely to lead to future developments in the lung cancer treatment field.

\section{ORCID}

Danbi Seo: https://orcid.org/0000-0002-9996-922X

Dain Kim: https://orcid.org/0000-0003-2512-2365

Yeonsoo Chae: https://orcid.org/0000-0003-2648-8238

Wanyeon Kim: https://orcid.org/0000-0002-7611-5248

\section{Authors' Contribution}

Conceptualization: WK. Data curation: DS, DK, WK. Formal analysis: DS, DK, WK. Methodology: DS, YC, WK. Writing original draft: DS, DK, YC, WK.

\section{Conflicts of Interest}

No potential conflicts of interest relevant to this article was reported.

\section{References}

1. Romaszko AM, Doboszynska A. Multiple primary lung cancer: a literature review. Adv Clin Exp Med 2018;27:725-730.

2. Collins LG, Haines C, Perkel R, Enck RE. Lung cancer: diagnosis and management. Am Fam Physician 2007;75:56-63.

3. Jacobsen MM, Silverstein SC, Quinn M, Waterston LB, Thomas CA, Benneyan JC, et al. Timeliness of access to lung cancer diagnosis and treatment: a scoping literature review. Lung Cancer 2017;112:156-164.

4. Hoy H, Lynch T, Beck M. Surgical treatment of lung cancer. Crit Care Nurs Clin North Am 2019;31:303-313.

5. Qian X, Zhao J, Yeung PY, Zhang QC, Kwok CK. Revealing lncRNA structures and interactions by sequencing-based approaches. Trends Biochem Sci 2019;44:33-52.

6. Nigita G, Marceca GP, Tomasello L, Distefano R, Calore F, Veneziano D, et al. ncRNA editing: functional characterization and computational resources. Methods Mol Biol 2019;1912:133174.

7. Anastasiadou E, Jacob LS, Slack FJ. Non-coding RNA networks in cancer. Nat Rev Cancer 2018; 18:5-18.

8. Paraskevopoulou MD, Hatzigeorgiou AG. Analyzing miRNA-lncRNA interactions. Methods Mol Biol 2016;1402:271-286.

9. Liu B, Li J, Cairns MJ. Identifying miRNAs, targets and functions. Brief Bioinform 2014;15:1-19.

10. Saliminejad K, Khorram Khorshid HR, Soleymani Fard S, Ghaffari SH. An overview of microRNAs: biology, functions, thera- 
peutics, and analysis methods. J Cell Physiol 2019;234:54515465.

11. Bak RO, Mikkelsen JG. miRNA sponges: soaking up miRNAs for regulation of gene expression. Wiley Interdiscip Rev RNA 2014; 5:317-333.

12. Vaschetto LM. miRNA activation is an endogenous gene expression pathway. RNA Biol 2018;15:826-828.

13. Acunzo M, Romano G, Wernicke D, Croce CM. MicroRNA and cancer: a brief overview. Adv Biol Regul 2015;57:1-9.

14. Hanahan D, Weinberg RA. Hallmarks of cancer: the next generation. Cell 2011;144:646-674.

15. Sandiford OA, Moore CA, Du J, Boulad M, Gergues M, Eltouky $\mathrm{H}$, et al. Human aging and cancer: role of miRNA in tumor microenvironment. Adv Exp Med Biol 2018;1056:137-152.

16. Iacona JR, Lutz CS. miR-146a-5p: Expression, regulation, and functions in cancer. Wiley Interdiscip Rev RNA 2019;10:e1533.

17. Bica-Pop C, Cojocneanu-Petric R, Magdo L, Raduly L, Gulei D, Berindan-Neagoe I. Overview upon miR-21 in lung cancer: focus on NSCLC. Cell Mol Life Sci 2018;75:3539-3551.

18. Shen YY, Cui JY, Yuan J, Wang X. MiR-451a suppressed cell migration and invasion in non-small cell lung cancer through targeting ATF2. Eur Rev Med Pharmacol Sci 2018;22:5554-5561.

19. Jiang YJ, Bikle DD. LncRNA profiling reveals new mechanism for VDR protection against skin cancer formation.J Steroid Biochem Mol Biol 2014;144 Pt A:87-90.

20. Huang Y. The novel regulatory role of $\operatorname{lncRNA}$-miRNA-mRNA axis in cardiovascular diseases. J Cell Mol Med 2018;22:57685775.

21. Do H, Kim W. Roles of Oncogenic long non-coding RNAs in cancer development. Genomics Inform 2018;16:e18.

22. Ma Y, Zhang J, Wen L, Lin A. Membrane-lipid associated IncRNA: a new regulator in cancer signaling. Cancer Lett 2018; 419:27-29.

23. Wang X, Yin H, Zhang L, Zheng D, Yang Y, Zhang J, et al. The construction and analysis of the aberrant lncRNA-miRNA-mRNA network in non-small cell lung cancer. J Thorac Dis 2019; 11:1772-1778.

24. Seo D, Kim D, Kim W. Long non-coding RNA linc00152 acting as a promising oncogene in cancer progression. Genomics Inform 2019;17:e36.

25. Li JH, Liu S, Zhou H, Qu LH, Yang JH. starBase v2.0: decoding miRNA-ceRNA, miRNA-ncRNA and protein-RNA interaction networks from large-scale CLIP-Seq data. Nucleic Acids Res 2014;42:D92-D97.

26. Bhan A, Soleimani M, Mandal SS. Long noncoding RNA and cancer: a new paradigm. Cancer Res 2017;77:3965-3981.

27. Qi X, Zhang DH, Wu N, Xiao JH, Wang X, Ma W. ceRNA in can- cer: possible functions and clinical implications. J Med Genet 2015;52:710-718.

28. Liu Y, Xue M, Du S, Feng W, Zhang K, Zhang L, et al. Competitive endogenous RNA is an intrinsic component of EMT regulatory circuits and modulates EMT. Nat Commun 2019;10:1637.

29. Li DS, Ainiwaer JL, Sheyhiding I, Zhang Z, Zhang LW. Identification of key long non-coding RNAs as competing endogenous RNAs for miRNA-mRNA in lung adenocarcinoma. Eur Rev Med Pharmacol Sci 2016;20:2285-2295.

30. Zhao Y, Feng C, Li Y, Ma Y, Cai R. LncRNA H19 promotes lung cancer proliferation and metastasis by inhibiting miR-200a function. Mol Cell Biochem 2019;460:1-8.

31. Ren J, Fu J, Ma T, Yan B, Gao R, An Z, et al. LncRNA H19-elevated LIN28B promotes lung cancer progression through sequestering miR-196b. Cell Cycle 2018;17:1372-1380.

32. Liu L, Liu L, Lu S. IncRNA H19 promotes viability and epithelial-mesenchymal transition of lung adenocarcinoma cells by targeting miR-29b-3p and modifying STAT3. Int J Oncol 2019; 54:929-941.

33. Li S, Mei Z, Hu HB, Zhang X. The lncRNA MALAT1 contributes to non-small cell lung cancer development via modulating miR-124/STAT3 axis. J Cell Physiol 2018;233:6679-6688.

34. Wei S, Wang K, Huang X, Zhao Z, Zhao Z. LncRNA MALAT1 contributes to non-small cell lung cancer progression via modulating miR-200a-3p/programmed death-ligand 1 axis. Int J Immunopathol Pharmacol 2019;33:2058738419859699.

35. Zhen Q, Gao LN, Wang RF, Chu WW, Zhang YX, Zhao XJ, et al. LncRNA DANCR promotes lung cancer by sequestering miR216a. Cancer Control 2018;25:1073274818769849.

36. Wang M, Mao C, Ouyang L, Liu Y, Lai W, Liu N, et al. Long noncoding RNA LINC00336 inhibits ferroptosis in lung cancer by functioning as a competing endogenous RNA. Cell Death Differ 2019;26:2329-2343.

37. Liu H, Han L, Liu Z, Gao N. Long noncoding RNA MNX1-AS1 contributes to lung cancer progression through the miR-527/ BRF2 pathway. J Cell Physiol 2019;234:13843-13850.

38. Lu W, Zhang H, Niu Y, Wu Y, Sun W, Li H, et al. Long non-coding RNA linc00673 regulated non-small cell lung cancer proliferation, migration, invasion and epithelial mesenchymal transition by sponging miR-150-5p. Mol Cancer 2017;16:118.

39. Tang Y, Wu L, Zhao M, Zhao G, Mao S, Wang L, et al. LncRNA SNHG4 promotes the proliferation, migration, invasiveness, and epithelial-mesenchymal transition of lung cancer cells by regulating miR-98-5p. Biochem Cell Biol 2019;97:767-776.

40. Yang J, Lin X, Jiang W, Wu J, Lin L. IncRNA LEF1-AS1 promotes malignancy in non-small-cell lung cancer by modulating the miR489/SOX4 axis. DNA Cell Biol 2019;38:1013-1021. 
41. Nie W, Ge HJ, Yang XQ, Sun X, Huang H, Tao X, et al. LncRNA-UCA1 exerts oncogenic functions in non-small cell lung cancer by targeting miR-193a-3p. Cancer Lett 2016;371:99-106.

42. Li Z, Lu Q, Zhu D, Han Y, Zhou X, Ren T. Lnc-SNHG1 may promote the progression of non-small cell lung cancer by acting as a sponge of miR-497. Biochem Biophys Res Commun2018; 506:632-640.

43. Yu W, Sun Z, Yang L, Han Y, Yue L, Deng L, et al. lncRNA PTAR promotes NSCLC cell proliferation, migration and invasion by sponging microRNA101. Mol Med Rep 2019;20:4168-4174.

44. Imai D, Yoshizumi T, Okano S, Uchiyama H, Ikegami T, Harimoto $\mathrm{N}$, et al. The prognostic impact of programmed cell death ligand 1 and human leukocyte antigen class I in pancreatic cancer. Cancer Med 2017;6:1614-1626.

45. Ahn BC, Pyo KH, Xin CF, Jung D, Shim HS, Lee CY, et al. Comprehensive analysis of the characteristics and treatment outcomes of patients with non-small cell lung cancer treated with anti-PD-1 therapy in real-world practice. J Cancer Res Clin Oncol 2019; 145:1613-1623.

46. Liu C, Yang Z, Deng Z, Zhou Y, Gong Q, Zhao R, et al. Upregulated lncRNA ADAMTS9-AS2 suppresses progression of lung cancer through inhibition of miR-223-3p and promotion of TGFBR3. IUBMB Life 2018;70:536-546.

47. Acha-Sagredo A, Uko B, Pantazi P, Bediaga NG, Moschandrea C, Rainbow L, et al. Long non-coding RNA dysregulation is a frequent event in non-small cell lung carcinoma pathogenesis. $\mathrm{Br} \mathrm{J}$ Cancer 2020;122:1050-1058.

48. Ma J, Yan H, Zhang J, Tan Y, Gu W. Long-chain non-coding RNA (lncRNA) MT1JP suppresses biological activities of lung cancer by regulating miRNA-423-3p/Bim axis. Med Sci Monit 2019;25:5114-5126.

49. Hao XZ, Yang K. LncRNA MAGI2-AS3 suppresses the proliferation and invasion of non-small cell lung carcinoma through miRNA-23a-3p/PTEN axis. Eur Rev Med Pharmacol Sci 2019;23: 7399-7407.

50. Xia H, Xiu M, Gao J, Jing H. LncRNA PLAC 2 downregulated miR-21 in non-small cell lung cancer and predicted survival. BMC Pulm Med 2019;19:172.

51. Liu X, Ma J, Xu F, Li L. TINCR suppresses proliferation and invasion through regulating miR-544a/FBXW7 axis in lung cancer. Biomed Pharmacother 2018;99:9-17.

52. Li Y, Zhao L, Zhao P, Liu Z. Long non-coding RNA LINC00641 suppresses non-small-cell lung cancer by sponging miR-424-5p to upregulate PLSCR4. Cancer Biomark 2019;26:79-91.

53. Zhang G, Wang Q, Zhang X, Ding Z, Liu R. LncRNA FENDRR suppresses the progression of NSCLC via regulating miR-761/ TIMP2 axis. Biomed Pharmacother 2019;118:109309.
54. Zhuan B, Lu Y, Chen Q, Zhao X, Li P, Yuan Q, et al. Overexpression of the long noncoding RNA TRHDE-AS1 inhibits the progression of lung cancer via the miRNA-103/KLF4 axis. J Cell Biochem 2019; 120:17616-17624.

55. Ao X, Jiang M, Zhou J, Liang H, Xia H, Chen G. lincRNAp21 inhibits the progression of nonsmall cell lung cancer via targeting miR175p. Oncol Rep 2019;41:789-800.

56. Dong L, Li G, Li Y, Zhu Z. Upregulation of long noncoding RNA GAS5 inhibits lung cancer cell proliferation and metastasis via miR-205/PTEN axis. Med Sci Monit 2019;25:2311-2319.

57. Mei Y, Si J, Wang Y, Huang Z, Zhu H, Feng S, et al. Long Noncoding RNA GAS5 suppresses tumorigenesis by inhibiting miR-23a rxpression in non-small cell lung cancer. Oncol Res 2017;25: 1027-1037.

58. Xue Y, Ni T, Jiang Y, Li Y. Long noncoding RNA GAS5 inhibits tumorigenesis and enhances radiosensitivity by suppressing miR135 b expression in non-small cell lung cancer. Oncol Res 2017; 25:1305-1316.

59. Baskar R, Lee KA, Yeo R, Yeoh KW. Cancer and radiation therapy: current advances and future directions. Int J Med Sci 2012; 9:193-199.

60. Buckley AM, Lynam-Lennon N, O'Neill H, O'Sullivan J. Targeting hallmarks of cancer to enhance radiosensitivity in gastrointestinal cancers. Nat Rev Gastroenterol Hepatol 2020;17:298-313.

61. Lynch TJ, Bell DW, Sordella R, Gurubhagavatula S, Okimoto RA, Brannigan BW, et al. Activating mutations in the epidermal growth factor receptor underlying responsiveness of non-smallcell lung cancer to gefitinib. N Engl J Med 2004;350:2129-2139.

62. Dasari S, Tchounwou PB. Cisplatin in cancer therapy: molecular mechanisms of action. Eur J Pharmacol 2014;740:364-378.

63. Yang QS, Li B, Xu G, Yang SQ, Wang P, Tang HH, et al. Long noncoding RNA LINC00483/microRNA-144 regulates radiosensitivity and epithelial-mesenchymal transition in lung adenocarcinoma by interacting with HOXA10. J Cell Physiol 2019; 234:11805-11821.

64. Liu AM, Zhu Y, Huang ZW, Lei L, Fu SZ, Chen Y. Long noncoding RNA FAM201A involves in radioresistance of non-small-cell lung cancer by enhancing EGFR expression via miR-370. Eur Rev Med Pharmacol Sci 2019;23:5802-5814.

65. Zhang J, Li W. Long noncoding RNA CYTOR sponges miR-195 to modulate proliferation, migration, invasion and radiosensitivity in nonsmall cell lung cancer cells. Biosci Rep 2018;38:BSR2 0181599.

66. Chen ZY, Liu HY, Jiang N, Yuan JM. LncRNA HOST2 enhances gefitinib-resistance in non-small cell lung cancer by down-regulating miRNA-621. Eur Rev Med Pharmacol Sci 2019;23:99399946. 
67. Ma G, Zhu J, Liu F, Yang Y. Long noncoding RNA LINC00460 promotes the gefitinib resistance of nonsmall cell lung cancer through epidermal growth factor receptor by sponging miR-7695p. DNA Cell Biol 2019;38:176-183.

68. Wang L, Shang X, Feng Q. LncRNA TATDN1 contributes to the cisplatin resistance of non-small cell lung cancer through
TATDN1/miR-451/TRIM66 axis. Cancer Biol Ther 2019;20: 261-271.

69. Zeng F, Wang Q, Wang S, Liang S, Huang W, Guo Y, et al. Linc00173 promotes chemoresistance and progression of small cell lung cancer by sponging miR-218 to regulate Etk expression. Oncogene 2020;39:293-307. 